\section{Submitted from BMJ Quality}

\title{
Making the journey safe: recognising and responding to severe sepsis in accident and emergency
}

\author{
Sarah Pinnington, Brigid Atterton, Sarah Ingleby
}

To cite: Pinnington S, Atterton B, Ingleby S. Making the journey safe: recognising and responding to severe sepsis in accident and emergency. BMJ Quality Improvement Reports 2016;5:u210706.w4335 doi:10.1136/bmjquality. u210706.w4335

Received 13 June 2016 Revised 22 August 2016

\section{CrossMark}

Central Manchester University Hospitals NHS Foundation Trust, UK

Correspondence to Sarah Pinnington sarah. pinnington@cmft.nhs.uk

\section{ABSTRACT}

Severe sepsis is a clinical emergency. Despite the nationwide recognition of the sepsis six treatment bundle as the first line emergency treatment for this presentation, compliance in sepsis six provision remains inadequately low. The project goals were to improve compliance with the implementation of the Sepsis Six in patients with severe sepsis and/or septic shock. In improving timely care delivery it was anticipated improvements would be made in relation to patient safety and experience, and reductions in length of stay (LoS) and mortality. The project intended to make the pathway for those presenting with sepsis safe and consistent, where sepsis is recognised and treated in a timely manner according to best practice.

The aim of the project was to understand the what the barriers where to providing safe effective care for the patient presenting with severe sepsis in A\&E. Using the Safer Clinical Systems (SCS) tools developed byte Health Foundation and Warwick University, the project team identified the hazards and associated risks in the septic patient pathway. The level of analysis employed enabled the project team to identify the major risks, themes, and factors of influence within this pathway. The analysis identified twenty nine possible interventions, of which six were chosen following option appraisal. Further interventions were recommended to the accident and emergency as part of a business case and further changes in process.

Audits identified all severely septic patients presenting to A\&E in October $2014(\mathrm{n}=67)$ and post intervention in September $2015(n=93)$. Compared analysis demonstrated an increase in compliance with the implementation of the sepsis six care bundle from $7 \%$ to $41 \%$, a reduction in LoS by 1.9 days and a decrease in 30 day mortality by $50 \%$. Additional audit reviewed the management of 10 septic patients per week for the duration of the project to assess the real time impact of the selected interventions.

\section{PROBLEM}

Severe sepsis is a clinical emergency. Despite the nationwide recognition of the sepsis six treatment bundle as the first line emergency treatment for this presentation, compliance in sepsis six provision remains inadequately low. Clinical audits have shown low compliance with the sepsis six care bundle within the accident and emergency (A\&E) department on a national and local scale highlighting an urgent need for improvement. ${ }^{1}$ To understand the problem in detail an audit of all patients presenting to Central Manchester University Hospitals NHS Foundation Trust (CMFT) adult A\&E department with severe sepsis in October 2014. This audit identified that $7 \%$ of patients presenting with severe sepsis received the full sepsis six within one hour of arriving to the department, highlighted an urgent requirement for improvement.

CMFT\&s adult A\&E department is located within Manchester's city centre and the hospital catchment area ensures a wide variety of presentations among a diverse population. Daily attendances often reach 300 patients, which can create a busy and highly pressured working environment. While the project team understood that the environment and some staffing issues were factors that may have compounded the problem of inadequate recognition and management of severely septic patients, it was believed that systems and processes could be improved for the benefit of this patient group. In order to understand these systems and processes the project team utilised tools that were designed for the Health Foundation and Warwick University Safer Clinical Systems (SCS) project. ${ }^{2}$ The project aimed to increase compliance in the delivery of the Sepsis Six in A\&E to $50 \%$ within six month intervention time period. As there were no previous data on LoS and 30 day mortality before the initial audit, the overall aim was to compare the data from the initial and end audits from this project to provide a baseline for these figures.

\section{BACKGROUND}

Severe sepsis is a major healthcare issue in the UK with 100000 cases presenting to 
hospital in the UK per year. ${ }^{3}$ The mortality rate for patients presenting with severe sepsis has been estimated as between $28 \%$ and $50 \%$ in the UK, with an estimated 37000 patient deaths and a further 65000 people left with serious long term complications. ${ }^{4}$ The economic burden of this patient group upon the healthcare economy due to these failures is considerable, with an average cost of about $£ 200000$ per admission. This means that improved care could lead to the potential savings of $£ 196$ million a year per annum. ${ }^{3}$

The current Trust policy used at CMFT is based on systemic inflammatory response criteria (SIRS). The SIRS criteria was utilised by the team as the initial tool to identify the severely septic patients. The SIRS criteria were defined in 1992 as the clinical expression of the host response to inflammation. ${ }^{4}$ SIRS remained the predominant approach to screening and classifying sepsis, with two or more SIRS criteria being an indicator of a systemic response to infection. ${ }^{5}$ The Trust's pathway mirrors this approach to identify potentially septic patients.

Severe sepsis is a systemic response to infection leading to severe organ dysfunction which can ultimately lead to sepsis shock and death. ${ }^{6}$ The gold standard treatment is the completion of the sepsis six bundle within one hour of presentation. ${ }^{1}$ Research has shown that the timely completion of this treatment bundle improves patient outcomes, reduces LoS and mortality. ${ }^{3}{ }^{6}$ Despite the establishment of national campaigns to address this failure in care provision, national audits have highlighted severe deficiencies in delivery of care, ${ }^{16}$ and the immediate requirement for improvement in the recognition and delivery of care for this patient group has been highlighted as a national priority. ${ }^{6} 78$

\section{BASELINE MEASUREMENT}

For this project it was necessary to obtain a representational sample of patients presenting with sepsis at A\&E. It was decided that capturing all patients presenting with signs and symptoms of severe sepsis over a one month period would provide a data sample capable of detailed data analysis. This would firstly ascertain the performance of the department in the management of severely septic patients and secondly gain an informed idea of the actual number of severely septic that presented over this time period.

For these baseline figures, the project team proposed to identify all of the patients presenting with severe sepsis and septic shock in A\&E over the month of October 2014. The decision to use the Systemic Inflammatory Response Syndrome (SIRS) criteria as the first method of identifying the patient group was primarily lead by the Trust's sepsis guidelines, which applied SIRS as the first identifier of possible sepsis. SIRS data were collected from the Patientrack ${ }^{\mathrm{TM}}$ electronic clinical observation recording and alerting system currently used throughout the Trust. Laboratory results from the Trust's clinical work base system were used to collect details of blood glucose and white cell count results to complete the information required for the SIRS criteria. Together this generated a database which identified all patients that triggered SIRS in A\&E.

There was an obvious oversight during initial analysis in the use of clinical observations only in the identification of severe sepsis. Patients who presented in a critical condition and treated in the resuscitation area of the department were not consistently included in the database. Initial electronic submission of clinical observations were not always priority in these critical situations, leading to missed patients who may not trigger the SIRS criteria following treatment or those who had been rapidly transferred to critical care or theatre. To resolve these issues and ensure capture of all eligible patients, it was necessary to use other data sources. Data were obtained from the arterial blood gas machines in the department to identify patients with a lactate measurement $\geq 2 \mathrm{mmols} / \mathrm{L}$. Elevated lactate levels are a marker of cellular dysfunction invariably seen in severe sepsis. ${ }^{9}$ The use of elevated lactate measurements, alongside capturing all critical care admissions within 24 hours of A\&E presentation allowed the project team to identify patients potentially missed from the initial SIRS data.

The resulting baseline data found 948 patients met the set criteria. Case notes, laboratory test results, and clinical observations were reviewed by the project team to identify severe sepsis cases. In total 67 patients were deemed to be severely septic. Analysis found that only $10 \%$ of the 67 patients received the Sepsis Six care bundle within one hour of presentation and compliance with each element of the sepsis six need improvement (see supplementary file "baseline audit data")

\section{DESIGN}

The quality improvement project team consisted of a lead nurse for acute care, two nurse project facilitators, and an A\&E consultant. A number of tools were used to gain a greater understanding of the current process and areas of risk within the patient pathway. These tools were created and utilised in the SCS project in 2011 in conjunction with Warwick University and the Health Foundation. The tools enable the team to review the patient pathway and identify levels of risk. They have been successfully utilised before within CMFT and the agreement was these tools would enable effective analysis of the areas of risk that would ultimately lead to appropriate interventions. The initial tool utilised was the high level process map, to ensure the project team have an initial understanding of the process. The severely septic patient journey was documented in sequential order, from admission route to discharge. Areas of potential risk were identified throughout the journey; identifying barriers for the timely recognition and management of the severely septic patient. This high level of risk faced by the septic patient required further analysis to determine the necessary interventions to make the journey safe. 
Following the high level mapping, we invited a wider team of nurses and medics from the department and undertook two swim lane maps were made of different patient journeys; one a timely and appropriate response to sepsis, with the second being not an ideal or timely response. These swim lane maps identify all those involved in the journey and the details of the pathway, identifying where the process has not been as it should have been and again identifying bottle necks or areas of risk. This process again uncovered clear issues with the present process and by having the wider team present, began the initial engagement of the staff.

Following the identification of areas of risk/concern, the project team employed Failure Modes and Effects Analysis (FMEA) approach, which enabled a proactive systematic analysis of where processes involved in the septic patient's journey could go wrong. By utilising the knowledge gained from the high level process map and swim lane maps (as well as involving various levels of the A\&E team) it helped establish a shared understanding of risks, forming the basis for development of patient safety initiatives. This analysis was strengthened by undertaking Hierarchical Task Analysis (HTA). This human factors analysis allowed the identification of tasks and sub-tasks; each task and subtask was risk ranked, identifying where things go wrong, reviewing how they go wrong, and what influences what goes wrong. This examination of intrinsic and extrinsic influences allowed for the recognition of the necessary changes in process needed to improve the care of the septic patient in A\&E. Present mitigations were identified as well as a number of areas that were not presently as they should be and could be seen as potential interventions to reduce the risks.

In total there were twenty-seven improvement interventions that were generated in response to the areas of risk. An options appraisal was completed including ease of implementation, cost effectiveness, acceptability, effect on patient safety and more, with staff from the department to ascertain which interventions would be the most beneficial and achievable. The chosen interventions were grouped together into three themes:

Theme 1: Improving time to treatment; Theme 2: Improving communication; Theme 3: Improving awareness of sepsis in recognition and timely response.

\section{STRATEGY}

Plan, do, study, act (PDSA) cycles were used to trial the improvement interventions and assess the impact on the compliance with the Sepsis Six. Alongside the PDSA cycles there were a number of process and outcome measures identified to observe for impact of the interventions. The project team reviewed ten cases per week, allowing for continuous evaluation of the impact of interventions and the project to be reactive to any compliance issues identified.

PDSA 1: Identification of septic patient for was a time consuming task when first looking for the baseline data in October and it was obvious that a more effective way was required. Development of sepsis database was undertaken to identify all A\&E patients triggering SIRS, linking a positive result with early warning scores (EWS), microbiology and laboratory results indicating organ dysfunction. This enabled an improved approach to identification of severely septic patients, rather than manually searching through case notes and various clinical systems. The initial development of the database required a number of changes in regard to the information needed to ensure all patients were captured. The addition of critical care admission data also ensured that patients who may have been taken directly to theatre or critical care were not missed from the database.

The database has evolved further, creating an inpatients option. This allows specialties within the Trust to have the means to understand of the prevalence of sepsis presentation in their patient groups, and simplifies the process of identification of patients to audit for compliance with sepsis management.

PDSA 2: Continuing review of ten patient cases per week for the duration of the project was undertaken by the project facilitators. The number of 10 patients was specifically chosen as it would identify approximately half of the severely septic patients presenting at A\&E per week, based on the baseline data numbers and it was able to be managed by the project facilitators. These patients were chosen at random utilising the sepsis database. The process measures that we were recording were average time to the Sepsis Six elements from presentation at the A\&E department and overall compliance to the Sepsis Six for the review group were calculated weekly. This gave the opportunity for timely assessment of the impact of interventions and also allowed the project team to modify certain interventions in response to themes/issues highlighted by the review data. This data was displayed on the project boards in the department and feedback to all staff individually via email.

PDSA 3: Education of the A\&E team was a top priority to ensure that the staff were knowledgeable and up to date regarding sepsis recognition and management. To ensure the education programme was effective, a number of trial sessions were held with three groups of four staff members. Feedback helped to develop an effective and interactive teaching session, which demonstrated improved knowledge with the use of pre and post teaching multiple choice questions (MCQ). Weekly education sessions were held for all grades of staff in the department to attend. These included information on sepsis recognition and management but also looked at reasons why diagnosis of sepsis could be delayed or missed. This allowed staff discussion of their own experiences, helping to identify where potential new improvements could be made and also helped evolve the contents of the teaching session to meet the needs of the department. Real life cases from the department were used in the teaching to further highlight issues found and for the team to have a greater understanding 
of how the departmental human factors could influence care. MCQs were once again employed to appraise the impact of the teaching, allowing timely changes to the content/teaching style to ensure essential information was conveyed. The MCQ questions were based on recognition and treatment of sepsis, including patient case based scenario questions. The times for the sessions were displayed on the project information board and also communicated to the team on a regular basis. Again numbers of staff trained and MCQ scores were monitored as process measures. The MCQ data demonstrated an increase in correct answers in the post teaching answers by up to $47 \%$.

PDSA 4: In the initial analysis period of the project it was identified that barriers to productive communication influenced the recognition and timely treatment in severe sepsis. These potential communication failures ranged from poor communication due to staff not knowing which nurse/doctor was looking after the patient, to delays in treatment due to staff not communicating that treatment had been prescribed. The project team through observations and feedback from all grades of staff found that there were times when the staff did not know the skills or grade of other staff and also which staff were assigned to which patients. This obviously could have impacted on staff and patient safety. To improve the communication between the team it was decided to look at the introduction of a safety huddle at the start of shifts. A number of styles were investigated and trialed using feedback from the staff to guide the project team to formulate a set standard for the department. The introduction of the 'pre brief' comprised of a huddle in each area of the department at the start of shifts, allowed nursing and medical teams to be introduced to the name, role and relevant skills of each member of the team, such as if nurse was able to do venepuncture or administer intravenous medications. To support the introduction of pre briefs, name boards were introduced in the individual patient rooms which displayed information of the named nurse and consultant in charge of the patients care. This ensured the doctor or allied health professional knew the named nurse who they should communicated with in regards to the management plan and treatment prescribed for the patient. Compliance with the number of completed pre briefs and name boards was undertaken, alongside qualitative data from staff feedback and questionnaires to understand the impact on team communication.

PDSA 5: Staff engagement was achieved through staff meetings, handovers, teaching sessions, and email contact with the A\&E team to keep them informed and updated with the project and highlighting their hard work. Continued engagement with staff throughout the project empowered staff members to take the project on as their own and feel proud of their work. Regular contact with staff through various mediums was integral to maintain the high profile of the project and in doing so raising awareness and ownership of the interventions, thus reducing the risk of the hazards. Feedback from staff helped to guide the methods of utilised to ensure the all staff had access and understood the project aims and interventions.

PDSA 6: The Trust sepsis stickers were introduced into the A\&E department (see supplementary information "sepsis sticker"). Audit of septic patients within the Trust had revealed improved compliance in the completion of the Sepsis Six within one hour when the stickers were used. The aim was to introduce the stickers in to the department, to improve documentation and Sepsis Six compliance. The stickers were placed with the sepsis assessment forms to ensure they were available if sepsis was suspected. The stickers act as a prompt to ensure the risk of not giving the correct treatment in a timely manner is reduced. The sepsis stickers list the Sepsis Six and the practitioner prompted to acknowledge the time zero of the severe sepsis diagnosis and to date and time when each component has been achieved. It also has a reminder to inform the senior nurse and senior doctor, ensuring senior expertise at the earliest opportunity in the management of the patient. The stickers are implemented by the first practitioner seeing the patient, and encourage clear documentation and compliance with the sepsis six. The uptake and impact on compliance was measured with the weekly patient audits as part of the process measures and the end of project audit.

\section{RESULTS}

The compliance and average time to the elements of the Sepsis Six were monitored throughout the project, reviewing ten septic patients per week. The purpose of this was to allow for the impact of the interventions to be measured and acknowledged and give an indication as to the present level of safety in the pathway. The data found average time and overall compliance significantly improved over the project time frame (see supplemental information "project results").

In September 2015 the project team repeated the initial audit, once again identifying all those patients attending A\&E with severe sepsis/ septic shock. Ninety-three patients were identified as having severe sepsis using the same inclusion criteria as October 2014. There was variation in the audit group sizes in both audits $(n=26)$. To ensure no patients had been overlooked in the October data before the sepsis database had been created, the October 2014 data was rechecked using the newly developed database to help identify any missed patients. However, there were no missed patients identified when using the same data collection points as the September 2015 audit, meaning both audits represented the actual severely septic patients attending A\&E in the audited time frames.

Analysis demonstrated a positive impact on the recognition of the patient with severe sepsis and the compliance with the Sepsis Six care bundle, with a $34 \%$ increase of patients receiving the Sepsis Six treatment 
within one hour from time zero. For our outcome measures the comparison with the initial October 2014 data found a decrease in length of stay by 1.9 days and a $50 \%$ reduction in 30 day mortality in the September 2015 patient group. Our second outcome measure established that time from prescription to administration of antibiotics reduced from 45.5 minutes to 11.2 minutes following the project interventions.

\section{LESSONS AND LIMITATIONS}

As stated previously the Trust current sepsis policy is based on the SIRS criteria. However, reliance on SIRS criteria has been under great scrutiny by the international research body and there has been pressure to develop an improved screening tool for sepsis. ${ }^{5}$ The recent international consensus has replaced SIRS as the suggested screening criteria with the introduction of Sequential [Sepsis-related Organ Failure Assessment (SOFA) and qSOFA for use in out of hospital settings, emergency departments, or general hospital wards. ${ }^{10} 11$ This is due to its low sensitivity and specificity in identifying septic patients, ${ }^{12}$ with SIRS not being specific to infection, as indicated by the name it is a systemic inflammatory response which can be activated by a wide range of clinical presentations. ${ }^{13}$ These concerns over the low sensitivity of SIRS were seen in the in the data collection of this project, where excessive numbers of patients were identified as SIRS positive yet less than $10 \%$ actually had severe sepsis.

Additionally, there are a number of groups that have been shown to not fit the criteria, exemplifying the complexities of identifying sepsis, which were also witnessed during this project. An example of this is in the elderly population, where pyrexia is often blunted or absent and the elderly patient may present with non-specific symptoms like recurrent falls, confusion or new incontinence rather than classic SIRS. ${ }^{15}$ Other patient groups seen to have potential for atypical presentation of sepsis include the young population who have the reserve to compensate well for a period of time before declining rapidly, as well as immunocompromised patients where any presentation of feeling unwell should treated as a medical emergency due to potential neutropenic sepsis and high associated mortality due to delay in treatment. ${ }^{16} 17$ In addition certain medications and prehospital treatment may also mask signs of sepsis, such as beta-blockers, paracetamol and fluid boluses, relaying on good clinical assessment and communication to ensure this is evidenced. Recent research has also indicated that there is a number of patients who have SIRS negative sepsis again adding to the debate, and requirement for more sensitive screening pathway. ${ }^{10}$ The project team are awaiting the upcoming NICE guidance alongside recently published international guidance to support the development of an improved and safer screening process for the Trust.

There were notable issues with the collection of time to treatment data for the Sepsis Six for fluid balance and oxygen leading to difficulties in accurate timing of delivery. Oxygen is not commonly prescribed within $\mathrm{A} \& \mathrm{E}$ and the correct percentage/flow was not always documented correctly with the input of observations leading to potential inadequacies in the data. Improvements in documentation have been seen since the implementation of the education sessions and a Trust wide oxygen improvement project will further enforce these standards. Fluid balance documentation was also a concern with many patients having no fluid balance recorded whilst in the department. This lead to the overall compliance with the Sepsis Six mainly being reduced due to the failure to fluid balance. Discussions with the departmental leads acknowledged that improvement was necessary and nominated staff are conducting an A\&E based improvement project. A substantial increase in compliance has been seen in the continued monthly audit of severely septic patient care with approximately $70 \%$ of patients having completed fluid balance charts compared to the end of project data of $45 \%$ completion. Both the improvements in documentation of fluid balance and oxygen have been further encouraged by the addition of the sepsis sticker documentation within the department.

Recent studies looking at the management of sepsis have found concerns regarding the effectiveness of the early goal directed treatment (EGDT) - Sepsis Six. The Protocolised Care for Early Septic Shock (ProCESS) and Australasian Resuscitation in Sepsis Evaluation (ARISE) trials did not detect a significant reduction in sepsis mortality with the use of EGDT compared with usual care, ${ }^{18} 19$ leading to argument that this may be the end for EGDT role in the management of septic patients. However, there questions remain regarding the overall significance of the results, with all patients in ARISE receiving antibiotics before randomisation, and patients including the usual-care group received rapid fluid resuscitation during the first six hours after randomisation. Mortality rates in ProCESS and ARISE were all significantly lower than the rate in the control group of the original 2001 EGDT trial, ${ }^{20}$ confirming that rapid recognition, early antibiotics, and aggressive fluid resuscitation are the essence to more effectively treating sepsis. While there is further research/discussion regarding which initial interventions are required for the best outcome, the use of the Sepsis Six will remain gold standard until further guidance.

The time limitations of the project meant that a number of recommendations the project had highlighted through risk analysis could not be fully implemented. These interventions, if they had been implemented in the project time frame, could have contributed to further improved results. However, these interventions have had to be supported through business cases which have been authorised within the project time frame due to the monetary requirements and multi-professional input. The first of the in-progress recommendations concerns staffing levels. Through 
observations of the severely septic patient journey through the department, the project identified the Rapid Assessment Unit (RAU) as a high risk area. The present process of RAU is as follows; ambulances arrive and patient is assessed in by a triage nurse. The patient is then booked in by the reception staff. Initial investigations are then carried out following which the patient is moved to an appropriate area in the department and care is handed over. Currently the staffing in the RAU is one registered nurse (RN) and two nursing assistants (NA). There is an office for a receptionist within RAU, although this role is not always filled due to staffing limitations. The original template for the staffing of RAU includes a consultant or senior doctor. This allows for a rapid assessment by a decision making clinician and facilitates the timely recognition of the sick patient and prescription of appropriate treatments. However, this is not always possible due to medical staffing numbers.

The project team believe that due to the high volume of ambulances and the pressures of operating this gateway, a second $\mathrm{RN}$ would be highly beneficial for the care of patients and flow of the department. This would provide additional advice support in managing the patients, enable immediate prescribed treatments to be carried out without causing delay in triage/holding ambulances unnecessarily, and allow nurse to nurse handover to be given on transfer of patient. Observations within RAU found that nurse handover was compromised when RAU is staffed with only one nurse due to the pressures of ambulance arrivals and managing the patient care. It was found that only $10 \%$ of patients from the RAU were handed over to the receiving nurse in the department when there was one RAU nurse, whilst this handover rate increased to $60 \%$ when there were two RAU nurses. The observations also found delays in test and investigations due to the time taken to admit a patient on to the electronic systems if there was not a receptionist in the RAU. To highlight these findings, data gathered were presented in a report to the senior management team. This report is currently being used as part of a larger business case to request for increased staffing and a review of the process.

The next in-progress recommendation looks at the provision of arterial blood gas analysers in the department. Currently there is only one arterial blood gas analyser in the department which is located in the resuscitation area. The project team has recommended that a second blood gas analyser would be beneficial due to the high volume of blood gases required, the pressure that this puts on the one current machine, and to support the layout of the department. Staff feedback and delays in lactate measurement highlight the risk of the current equipment, not only in the identification of sepsis but in the rapid identification of the sick patient. Data gathered by the project have been added to a business case for the purchase of a second blood gas analyser within the department.
Finally, the third in-progress recommendation concerns the introduction of needle free antibiotics, which allow for reconstitution of the antibiotic via an ampule of the drug pre-attached to the diluent bag. A pilot for needle free antibiotics had previously been conducted, demonstrating improved times of antibiotic administration which would be hugely beneficial in the A\&E department. This is presently under review.

The impact of education saw significant benefits in the timely recognition and management of severely septic patients within the A\&E department, with the average improvement of $93 \%$ in the correct answers in the pre and post training multiple choice questions. The project result process measure run charts, illustrate the positive impact the teaching had on the average time to elements of the Sepsis Six, with improvements seen after the implementation of teaching programmes. The delivery of standardised education sessions supported a consistent and knowledgeable approach to recognising, escalating, and delivering timely and appropriate management in severe sepsis. The sessions allowed the opportunity for further discussion and engagement with staff, embedding the project aims and allowing the project team to be responsive to any knowledge gaps or process issues. The fact that this increased sepsis based education had positively influenced compliance with the delivery of the Sepsis Six; the project team understood the importance to replicate the education throughout the Trust. Improved sepsis education is now received on induction for all grades of healthcare staff and is included on the yearly clinical mandatory training to ensure all clinical staff within the Trust are up to date and aware of the sepsis pathway. Simulation sessions have also been started and additional training is undertaken by sepsis champions that have been identified within the A \& E department to ensure sustainability.

\section{CONCLUSION}

The project team was able to identify the hazards within the septic patient journey using the Safer Clinical Systems approach, developing interventions that addressed these hazards to reduce the risk. The project has enabled the risks to be reduced (as is evident from the September audit) but the project team acknowledge that continued work is required to ensure an improved level of safety is achieved. To sustain the continued improvement and prevent a drift into failure the project team has appointed sepsis champions in the team to continue to drive the project aims and develop a teaching rota to ensure this initiative continues. This is also supported by continuing audit of the management of twenty septic patients per month to ensure compliance with the standards continues and that the department can be proactive in its approach to addressing any issues in a timely manner.

Due the significant results gained by this project, it is necessary to ensure these improvement methodology 
and interventions are shared throughout the Trust and further afield as necessary. The project team have begun supporting sepsis improvement projects with the Trust obstetrics team and enabling divisions to understand their sepsis management through audit supported by the development of the sepsis database. Further work is underway with the development of educational videos for public and staff to enhance awareness within the hospital and community settings.

Acknowledgements All the A\&E staff for their involvement and contributions to this project.

Declaration of interests Nothing to declare.

Ethical approval This work is deemed to be an improvement study and not a study on human subjects, therefore local policy excludes this from requiring ethical approval.

Open Access This is an open-access article distributed under the terms of the Creative Commons Attribution Non-commercial License, which permits use, distribution, and reproduction in any medium, provided the original work is properly cited, the use is non commercial and is otherwise in compliance with the license. See:

- http://creativecommons.org/licenses/by-nc/2.0/

- http://creativecommons.org/licenses/by-nc/2.0/legalcode

\section{REFERENCES}

1. CEM clinical audits 2011-2012: severe sepsis and septic shock. London: College of Emergency Medicine; 2012.

2. Heath Foundation (2009) Safer Clinical Systems. London: The Health Foundation. .

3. Health Service Ombudsman for England (2013). Time to act severe sepsis: rapid diagnosis and treatment saves lives. London: Parliamentary and Health Service Ombusdman.

4. Angus DC, Van Der Poll T. Severe Sepsis and Septic Shock. NEJM 2013; 369:840-85.

5. Bone R. Definitions for sepsis and organ failure. Crit Care Med 1992;20:724-6.
6. Daniels R, Nutbeam T, McNamara G, et al. The sepsis six and the severe sepsis resuscitation bundle: a prospective observational cohort study. EMJ 2011;28:507-12.

7. Kumar A, Roberts D, Wood KE, Light B, Parrillo JE, Sharma S, et al. Duration of hypotension before initiation of effective antimicrobial therapy is the critical determinant of survival in human septic shock. Crit Care Med 2016;34:1589-96.

8. NCEPOD (2015) Just Say Sepsis! A review of the process of care received by patients with sepsis. London: NCEPOD.

9. NHS England (2014) Improving outcomes for patients with sepsis. London: NHS England.

10. NHS England (2015) Commissioning for Quality and Innovation (CQUIN). London: NHS England.

11. Kaukonen KM, Bailey M, Pilcher J, Bellomo R. Systemic Inflammatory Response Syndrome Criteria in Defining Severe Sepsis. NEJM 2015; 372:1629-38.

12. Singer M, Deutschman CS, Seymour C, et al. The Third International Consensus Definitions for Sepsis and Septic Shock (Sepsis-3). JAMA 2016;315:801-10. doi:10.1001/jama.2016.0287.

13. Liao M, Lezotte D, Lowenstein SR, Howard K, Finley Z, Feng Z, Byyny BA, et al (2014) Sensitivity of systemic inflammatory response syndrome for critical illness among ED patients. Am J Emer Med; 32:1319-25.

14. Brun-Buisson $\mathrm{C}$. The epidemiology of the systemic inflammatory response. Intens Care Med 2000;S64-S74.

15. Opal SM, Girard TD, Ely EW. The immunopathogenesis of sepsis in elderly patients. Clin Infect Dis 2005;41:S504-12.

16. Clarke RT, Bird S, Kakuchi I, Littlewood TJ, Van Hamel Parsons V The signs, symtoms and help-seeking experiences of neutropenic sepsis patients before they reach hospital: a qualitative study. Support Care Cancer 2015; 23: 2687-94.

17. NICE guidelines [CG151]. Neutropenic sepsis: prevention and management in people with cancer. 2012. (Accessed 16 November 2015).

18. Peake SL, Bailey M, Bellomo R, Cameron PA, Cross A, Delaney A, Finfer S, Higgins A, Jones DA, Myburgh JA, Syres GA, Webb SA, Williams P. Australasian resuscitation of sepsis evaluation (ARISE): a multicentre, prospective, inception cohort study. Resuscitation 2009;80:811-8.

19. ProCESS investigators. A randomized trial of protocol-based care for early septic shock. NEJM 2014;370:1683-93.

20. Rivers E, Nguyen B, Havstad S, Ressler J, Muzzin A, Knoblich B, Peterson E, Tomlanovich M. Early Goal-Directed Therapy in the Treatment of Severe Sepsis and Septic Shock. NEJM 2001;345:1368-77. 